\title{
UIs Automatically Optimized for Your Smartphone
}

\author{
Hermann Kaindl \\ Vienna University of Technology \\ Gußhausstr. 27-29, A-1040 Vienna, Austria \\ kaindl@ict.tuwien.ac.at
}

\begin{abstract}
Graphical user interfaces (UIs) for PCs will most likely not fit relatively small screens of devices like today's Smartphones. Providing dedicated UIs for several devices manually, however, is costly and takes time. Therefore, we have developed an approach to (semi-)automatic generation of UIs for various devices. A designer defines classes of dialogues in a deviceindependent discourse model. Such a discourse model can be also viewed as specifying classes of scenarios, i.e., use cases. It refers to a domain model that specifies the domain-of-discourse of the dialogues between user and application. From such models, we can generate UIs (semi-)automatically. Recently, we included in this generation process automatic optimization based on heuristic search. In effect, this tutorial shows that and how user interfaces can be automatically optimized for your Smartphone.
\end{abstract}

Keywords: (Semi-)automatic generation of user interfaces, automatic optimization for small devices, Smartphones.

\section{Tutorial Goals}

The main goals are to show that usable user interfaces (UIs) can be generated (semi)automatically, in particular through automatic optimization based on heuristic search. This approach is particularly useful for optimizing UIs for relatively small screens like those of current Smartphones. Just based on a simple device specification of the Smartphone, the UI is specifically optimized for it. Such UIs can be generated fully automatically and optimally fit the given device, so that they are ready for real-world use, e.g., on Smartphones.

\section{Key Learning Outcomes}

In this tutorial, participants learn about modeling discourses using a new approach inspired by human-human communication. They will know how modeling discourses and generating user interfaces can be approached systematically. They will also see how techniques from heuristic search can be used for optimizing automatically generated user interfaces for small devices like today's Smartphones.

\section{$3 \mathrm{CV}$ of the Presenter}

Hermann Kaindl is the director of the Institute of Computer Technology at the Vienna Univ. of Technology. He joined this institute in early 2003 as a full professor. Prior to 
moving to academia, he was a senior consultant with the division of program and systems engineering at Siemens AG Austria. There he has gained more than 24 years of industrial experience in software development and human-computer interaction. $\mathrm{He}$ has published four books and more than a hundred papers in refereed journals, books and conference proceedings. He is a Senior Member of the IEEE, a Distinguished Scientist member of the ACM, a member of the INCOSE and the AAAI, and is on the executive board of the Austrian Society for Artificial Intelligence.

\section{References}

1. Bogdan, C., Falb, J., Kaindl, H., Kavaldjian, S., Popp, R., Horacek, H., Arnautovic, E., Szep, A.: Generating an Abstract User Interface from a Discourse Model Inspired by Human Communication. In: Proceedings of the 41st Annual Hawaii International Conference on System Sciences (HICSS-41). IEEE Computer Society Press, Big Island (2007)

2. Bogdan, C., Kaindl, H., Falb, J., Popp, R.: Modeling of interaction design by end users through discourse modeling. In: Proceedings of the 2008 ACM International Conference on Intelligent User Interfaces (IUI 2008). ACM Press, Maspalomas (2008)

3. Falb, J., Kaindl, H., Horacek, H., Bogdan, C., Popp, R., Arnautovic, E.: A discourse model for interaction design based on theories of human communication. In: CHI 2006 Extended Abstracts on Human Factors in Computing Systems, pp. 754-759. ACM Press, New York (2006)

4. Falb, J., Kavaldjian, S., Popp, R., Raneburger, D., Arnautovic, E., Kaindl, H.: Fully Automatic User Interface Generation from Discourse Models. In: Proceedings of the 2009 ACM International Conference on Intelligent User Interfaces (IUI 2009). ACM Press, Sanibel Island (2009); Tool demo paper

5. Falb, J., Popp, R., Röck, T., Jelinek, H., Arnautovic, E., Kaindl, H.: UI Prototyping for Multiple Devices Through Specifying Interaction Design. In: Baranauskas, C., Abascal, J., Barbosa, S.D.J. (eds.) INTERACT 2007. LNCS, vol. 4662, pp. 136-149. Springer, Heidelberg (2007)

6. Kaindl, H.: A Design Process Based on a Model Combining Scenarios with Goals and Functions. IEEE Transactions on Systems, Man, and Cybernetics (SMC) Part A 30, 537-551 (2000)

7. Kaindl, H., Jezek, R.: From Usage Scenarios to User Interface Elements in a Few Steps. In: Proceedings of the Fourth International Conference on Computer-Aided Design of User Interfaces (CADUI 2002), Valenciennes, France, pp. 91-102. Kluwer Academic Publishers, Dordrecht (2002)

8. Kavaldjian, S., Bogdan, C., Falb, J., Kaindl, H.: Transforming Discourse Models to Structural User Interface Models. In: Giese, H. (ed.) MODELS 2008. LNCS, vol. 5002, pp. 77-88. Springer, Heidelberg (2008)

9. Raneburger, D., Popp, R., Kavaldjian, S., Kaindl, H., Falb, J., Optimized, G.U.I.: Optimized GUI Generation for Small Screens. In: Model-Driven Development of Advanced User Interfaces, MDDAUI 2010 Workshop. Springer, Heidelberg (2011) 